\title{
INTEGRATED OPTICS IN ASTRONOMICAL INTERFEROMETRY
}

\author{
V. COUDÉ DU FORESTO \\ Observatoire de Paris, DESPA (CNRS URA 264), 92195 Meudon Cedex, France
}

\begin{abstract}
Integrated optical components (mostly single-mode fibers and couplers) can be used to achieve several functions that are needed in interferometry: coherent beam transportation and recombination, pathlength modulation and control for fringe tracking and double Fourier interferometry, spatial filtering of the wavefront and interferogram calibration. Their potential is assessed and the main problems encountered in their implementation are discussed: dispersion, polarization behavior, and especially starlight injection.
\end{abstract}

Key words: Stellar interferometry - Integrated optics - Single-mode fibers and couplers

\section{Introduction}

Waveguides have long been used in radio interferometry to transport radiation from the focus of the antennae to the correlator, but it was not until the last decade that appeared the first discussion of the use of fibers for optical interferometry (Froehly 1981). At first fibers were seen mainly as an elegant alternative to mirror trains for carrying light along complicated paths, and a way to relax the mechanical constraints on telescope positioning in space interferometers (Connes et al. 1984). Progressively the commercial availability of single-mode directional couplers and other components has broaden the scope of application of guided optics, and it is now realized that they can be employed to perform other functions that are needed in interferometry, such as beam transportation from the telescope foci to the recombination lab, beam recombination, spatial filtering, and pathlength modulation and control.

\section{Beam transportation}

The most important requirement that is put on a waveguide for interferometry is that it must preserve the phase information of the radiation. This is most commonly achieved with single-mode fibers, since then the propagation constant is in principle unique and well defined (even though broadband fringes can still be observed with few-mode fibers, provided the modes are separated at the output and recombined one by one (Shaklan 1990)).

Single-mode (SM) fibers differ in many ways from the large multimode fibers used in other astronomical instrumentation such as multi-object or imaging spectroscopy. Although the cladding has roughly the same diameter (typically $125 \mu \mathrm{m}$ ) their core is much smaller ( 3 to $9 \mu \mathrm{m}$ ), and because of that small size geometric optics cannot offer a correct description of their properties. The guided beam is not confined to the core and extends well into the cladding, which may in some circumstances carry the major part of the energy. A numerical aperture is defined $\left(N A=\sqrt{n_{\text {core }}^{2}-n_{\text {clad }}^{2}}\right)$ that expresses the index difference between the core and the cladding, but cannot be related to an acceptance angle for the rays. The most important parameter is the normalized frequency $V=2 \pi a N A / \lambda$, where $a$ is the core radius. Single-mode operation is achieved when $V<2.405$, and the mode profile of a step index fiber can be described as a function of $V$ only (Gloge 1971). An 
extensive monograph and a large bibliography on SM fibers and components was written by Neumann (1988).

An important feature of a single-mode fiber is that the output beam profile is totally independent from the launching conditions. In other words, the waveguide loses memory of the shape of the corrugated input wavefront to deliver at the output a spatially coherent, fixed beam with a constant (Gaussian-like) profile determined by the characteristics of the fundamental mode. In doing so, the fiber acts like an ideal spatial filter, a point most useful in interferometry as will be discussed below. When testing or aligning an instrument, the spatial coherence of the beam emitted by a single-mode fiber makes it a very attractive alternative to a pinhole wherever an unresolved point source is necessary. But because the far field diffraction profile is also quasi-Gaussian the beam cannot be entirely collected by a lens to be focused onto a detector. In a typical fiber, output optics with an f-ratio of 2 or faster are necessary to collect $90 \%$ of the energy or more (Coudé du Foresto and Mazé 1990).

\subsection{Polarization}

Ideally, in a perfect fiber the fundamental mode is degenerate for the two spatially orthogonal polarizations. Circular fibers in real life behave in a much different manner and exhibit birefringence and mode coupling. Unwanted birefringence occurs in non-perfect fibers because of random little shape and stress anisotropies. Even if it were perfectly manufactured, a circular-core fiber would not be neutral, except when kept straight. Otherwise, bending (Payne et al. 1982, Ross 1984, Ulrich et al. 1980), twisting (Payne et al. 1982) and stretching (Forman and Jahoda 1988) lead to additional birefringence.

In a perfect circular core fiber, a linearly polarized light will emerge as a linearly polarized light with the same orientation. In reality, the output state of polarization is generally an ellipse. However, Ramaswamy et al. (1978) have shown that for a fiber of any given length, excitation conditions exist at the input that result in a linearly polarized output.

If there is birefringence but no mode coupling, each polarization gives its own full visibility fringe pattern, but the two fringe patterns are not registered and the total visibility is decreased. In addition, the polarizations do not rotate. Thus, by placing a single polarizer at the input (or at the output) a maximum fringe contrast is insured. If there is mode coupling but no birefringence, each mode is contaminated by incoherent light coming from the other mode. The two fringe patterns are well registered but their individual visibility is not maximum. As both phenomena simultaneously occur a polarizer is helpful but does not ensure maximum contrast. Fortunately the state of polarization at the fiber output changes very little over periods of 24 hours or more (Harmon 1982), and the visibility loss due to polarization can be considered as part of the instrument transfer function and calibrated as such on an unresolved source.

The best way to control polarization is to use polarization maintaining, highly birefringent fibers. Polarization preserving fibers have a strong intrinsic birefringence that overwhelms the random birefringence, and well-defined principal axes; they maintain the polarization of propagating light even when the fiber is bent 
or twisted. The birefringence is usually induced by a shape anisotropy (elliptical or rectangular core) or a stress anisotropy (Panda, bow-tie fibers) if the circular geometry of the core needs to be maintained. At the output the two polarizations can be observed either separately or simultaneously if an adequate compensator is introduced. One can refer to Noda et al. (1986) for an overview of polarization preserving fibers with a large bibliography.

\subsection{DisPERSION}

In addition to the traditional overall pathlength equalization of an interferometer, material dispersion in the waveguide requires the physical length traveled by the guided light to be the same for both arms. Then in a wideband instrument, the total pathlength is equalized simultaneously for all wavelengths and the zero-order fringes add up constructively.

If the waveguide is longer on one side the zero-order fringes occur at various locations, and the modulation depth (defined as $I_{\max }-I_{\min } / I_{\max }+I_{\min }$ ) of the central fringe is decreased. Shaklan and Roddier (1987) have computed the loss of modulation depth with silica fibers for different bandpasses in the visible. A $0.5 \mathrm{~mm}$ fiber length difference causes a $20 \%$ loss in a wideband instrument $(680 \mathrm{~nm}$ $<\lambda<1060 \mathrm{~nm}$ ). It is possible to cut fibers to equal lengths within $10 \mu \mathrm{m}$, as was demonstrated by Burnett and Jones (1992) on $6 \mathrm{~m}$ long fibers. In the infrared, the equalization does not need to be as good since the bandpass is limited by the atmospheric windows and the intrinsic dispersion of the fluoride glass employed for IR fibers is lower than in silica (Jinguji et al. 1982).

The interferogram can also be recorded as a long scan around both sides of the zero optical pathlength difference, rather than just looking at the modulation of the central fringe. In this procedure, known as double Fourier interferometry (Mariotti and Ridgway 1988), the temporal and spatial coherence of the radiation are simultaneously probed to yield both spatial and spectral information on the source. Dispersion does not change the modulus of the fringes in the Fourier transform of the interferogram, but only their phase. It can be integrated in the imaginary part of the instrument transfer function and calibrated on a reference star if the waveguide length difference is stable in time. This might require an active thermal control of the fibers, for which a practical solution has been described by Connes and Reynaud (1988).

\subsection{COMPONENTS AVAILABILITY AND TRANSPARENCY}

Currently single-mode fibers are available for wavelengths ranging from the blue end of the visible to the mid infrared. In the visible and the near infrared $(480 \mathrm{~nm}<\lambda<$ $1800 \mathrm{~nm}$ ), fused silica fibers have been developed for high rate telecommunication applications and a wide range of components are available ${ }^{1}$. In current silica fibers

\footnotetext{
${ }^{1}$ In addition to purely integrated components, a certain number of hybrid systems have been developed that can also be useful in interferometry. They are usually optimized for the most common wavelengths used in telecommunications $(830,1064,1300$ and $1550 \mathrm{~nm})$, and include polarizers/depolarizers, multichannel switchers, filters, etc...
} 
the absorption is close to the theoretical minimum and strongly color dependent. For a $100 \mathrm{~m}$ long waveguide, typical transmissions (excluding Fresnel losses at each end) range from $50 \%$ at $480 \mathrm{~nm}$ to $98.9 \%$ at $1300 \mathrm{~nm}$. The transmission of polarization maintaining fibers, however, is usually not as good (from $10 \%$ in the blue to $95 \%$ in the near IR).

SM fibers in the infrared $(1.5 \mu \mathrm{m}<\lambda<4 \mu \mathrm{m})$ are more recent and made of fluoride glass (Monerie et al. 1985). In fluoride glass, the absorption minimum occurs around $2.6 \mu \mathrm{m}$ and with the current technology transmissions of at least $60 \%$ for $100 \mathrm{~m}$ can be achieved in the $\mathrm{K}$ band (with ample margin for improvement since the theoretical absorption minimum is around $0.001 \mathrm{~dB} / \mathrm{km}$ ). Polarization preserving fibers in the infrared are still mostly experimental.

\section{Beam recombination}

Coherent beam recombination can be done in a single-mode directional coupler, which performs the same function as a beamsplitter. Unlike a beamsplitter, however, the coupler is small, rugged, and virtually impossible to misalign. Its connection to a single-mode fiber or another coupler is straightforward and can be done in seconds if it is properly connectorized.

The basic component is the X-coupler that features two fiber inputs and two fiber outputs. If one input is used, the coupler distributes the signal between the two outputs. If both inputs are utilized, the coupler acts like a correlator between the two incoming beams. Coupling ratios are usually $50 / 50$ but can be made at different values, depending on applications. Polarization preserving couplers are also manufactured. Another component using polarization preserving fibers is a coupler that separates the two orthogonal polarizations. In addition to X-couplers that are commercially produced, single-mode star couplers with up to $10 \times 10$ terminals have also been made (Sheen and Giallorenzi 1979, Sheen 1980, Wang et al. 1985).

When recombining beams from three or more telescopes, integrated couplers offer a substantial simplification over an all-mirror solution. Several architectures for multiple beam correlation using two or more stages of X-couplers have been discussed (Léna et al. 1988, Shaklan 1989). Because connectorized components are easily plugged and unplugged, the correlator can be reconfigured quickly, giving an unmatched flexibility to the system. Current state of the art single-mode connectors have a typical insertion loss of $9 \%$ and can sustain over 1000 manipulations.

The potential of multiple beam correlation using directional couplers is illustrated in a laboratory experiment performed by Shaklan (1989). A 5-telescope interferometer was built with a correlator made of $6 \mathrm{X}$-couplers arranged in two stages; real image reconstruction could be produced from the simultaneous measurement of the complex visibilities at each of the array's ten baselines.

One can refer to Tekippe and Willson (1985) for a general review of single-mode directional couplers. The two most commonly used techniques for their fabrication are polishing and fusion-tapering. In polished couplers, the fibers are polished and held together with the two fiber cores running in parallel very close to each other along a few centimeters, so that mixing of the guided radiations can occur by coupling of the evanescent fields in the cladding. In taper-fused couplers, the fibers 
are twisted together, heated, fused and stretched. This is the preferred technique for a flattened wavelength response. On the other hand, polished couplers can more easily be tuned to a required coupling ratio at the nominal wavelength (Neumann 1988).

Important parameters for directional couplers in astronomical interferometry are their intrinsic loss (typically $2 \%$ or less for a fused X-coupler), phase dispersion and wavelength response. For example, the best silica wideband X-couplers offer a splitting ratio within the $40 / 60$ boundaries for an operating range $\delta \lambda / \lambda=0.3$. Currently available fluoride glass couplers are polished and their response is more chromatic (from $25 / 75$ to $75 / 25$ between 2 and $2.4 \mu \mathrm{m}$ ).

\section{Spatial filtering and visibility calibration}

One of the most interesting features of single-mode integrated optics is, through the perfect spatial filtering of the incoming wavefront, the possibility it offers to easily calibrate the fringe visibilities for each interferogram and obtain seeing independent, high precision estimates of the contrast.

In a classical interferometer, the observed fringe contrast can be expressed as the product of three quantities:

$$
\Gamma_{o b s}=V \times T_{i} \times T_{a},
$$

where $V$ is the object visibility at the sampled spatial frequency, $T_{i}$ the instrument transfer function and $T_{a}$ the atmospheric transfer function. $T_{i}$ is in principle constant and, in absence of turbulence, can be calibrated on a point source for which $V=1$ at every spatial frequency. The random seeing term $T_{a}$ mainly expresses the loss of transverse spatial coherence on the pupil. To know it with a good accuracy for each interferogram would require a complete wavefront sampling, a task that is expensive both in hardware and photons.

The situation is different in an interferometer using single-mode integrated optics, where the transverse coherence of the beam is forced by the waveguide. All turbulent modes of the atmosphere (except the piston mode that does not contribute to the loss of spatial coherence of the beam) are filtered out by the fiber. Seeing induced loss of contrast still occurs, because coupling fluctuations into the fiber destroy the intensity balance between the two arms of the interferometer. This is easily modeled and Eq. (1) can be rewritten:

$$
\Gamma_{o b s}=V \times T_{i} \times \frac{2 \sqrt{E F}}{E+F}
$$

where $E$ and $F$ are the relative intensities in each arm of the instrument. But here it should be noticed that the term $\frac{2 \sqrt{E F}}{E+F}$ is little seeing sensitive. For example, in the case of a coupling 2 times better in one arm than in another, the contrast is degraded by only $6 \%$. In addition, $E$ and $F$ are mere intensities that can easily be measured with the interferogram. It is then possible to correct a posteriori for the factor $\frac{2 \sqrt{E F}}{E+F}$ and obtain a seeing free estimate $\Gamma$ of the fringe contrast.

Figure 1 illustrates the concept of a stellar interferometer using integrated optics. Two telescopes sample the wavefront and inject starlight into a single-mode fiber; 


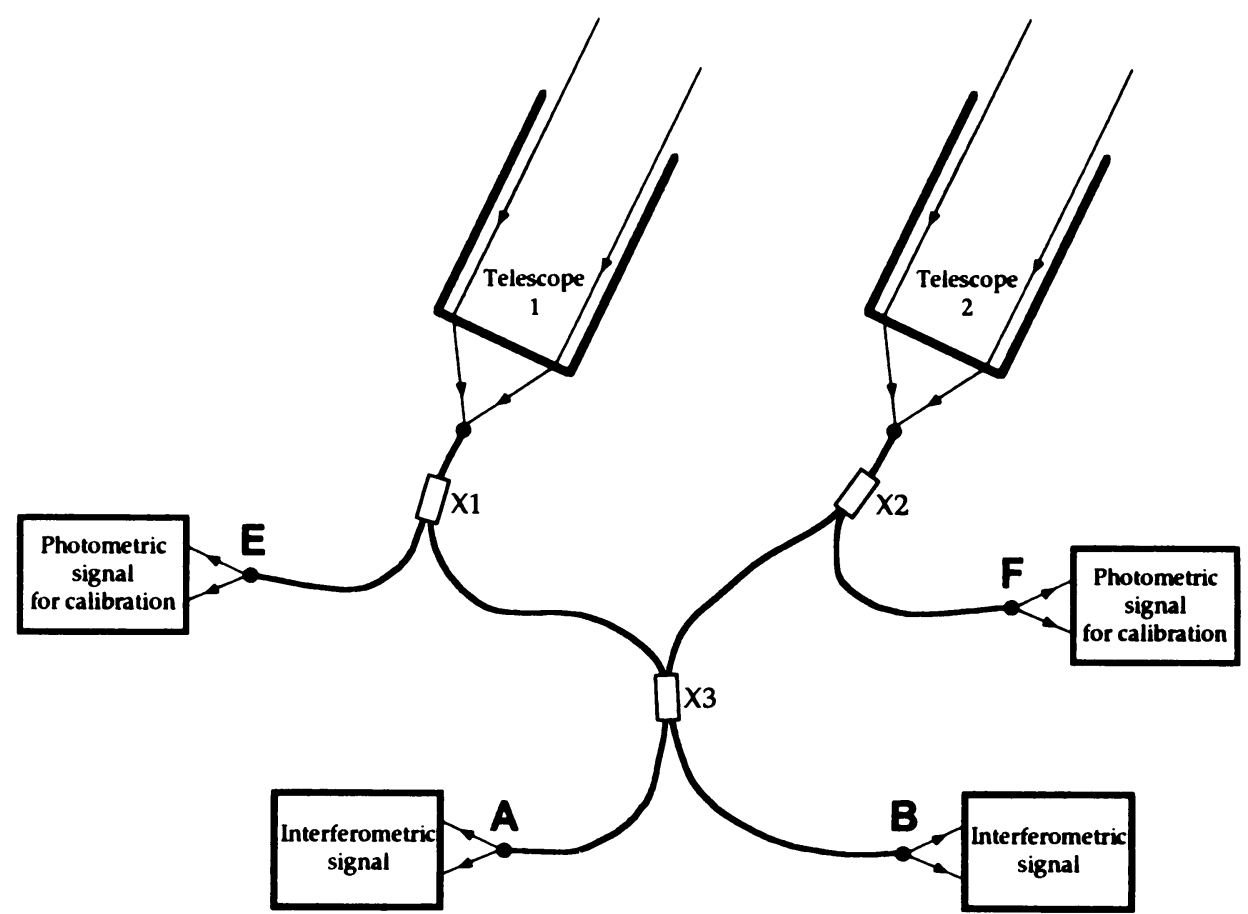

Fig. 1. Conceptual design of a stellar interferometer using integrated optics

coherent recombination is achieved within the $\mathrm{X} 3$ coupler, which produces in $\mathrm{A}$ and $\mathrm{B}$ the two complementary interferometric outputs of the system. Auxiliary couplers $\mathrm{X} 1$ and X2 extract a portion of the light in each arm of the interferometer to monitor the coupling fluctuations. The calibration signals observed in $\mathrm{E}$ and $\mathrm{F}$ are used in the data reduction process to correct the interferograms from seeing effects. The prototype FLUOR (Coudé du Foresto and Ridgway 1991) is an interferometric recombination unit that borrowed this concept to link two independent telescopes. With it, accuracies better than $1 \%$ have been demonstrated in the measurement of stellar fringe visibilities (Coudé du Foresto et al. 1992).

\section{Pathlength modulation and control}

Stretching a fiber provides an efficient way to control the optical pathlength in an interferometer. The fiber can either be stretched between two endpoints glued on a lever arm (Shaklan 1989), or wrapped onto a cylinder made of a piezo-electric ceramic that can expand or contract diametrically when a voltage is applied. The later solution has the advantage that the strain is regularly distributed on the fiber. Such an arrangement is cheap and, because no moving parts are involved, the response of the system can be extremely fast $(>1 \mathrm{kHz})$.

The main applications of this technique lie where a rapid control of short pathlength differences are needed: for example in fringe tracking (Rohloff and Leinert 
1991) and double Fourier interferometry (Zhao et al. 1992). Stretching a fiber may cause unwanted dispersion and polarization effects which are discussed in Zhao et al. (1992).

The stroke is limited by the size of the piezo cylinder (which governs the length of fiber that can be wrapped on it) and its expansion capability, rather than the expansion capability of the fiber itself (which is $2 \%$ or more before breakage). At present, a few hundred turns can be wrapped onto a piezo with a diameter of $6 \mathrm{~cm}$, for a maximum stroke of several $\mathrm{mm}$. Because that range is limited, it appears that the only function that cannot be performed by guided optics in a stellar interferometer is the pathlength compensation provided by a delay line. Even if one could wrap several kilometers of fiber on a cylinder and obtain a maximum expansion of $10 \mathrm{~m}$ or more, the dispersion induced by the extra length of waveguide would be prohibitive.

An alternative solution to build an all-fiber interferometer is to eliminate the need for a delay line by locating all the telescopes near the same wavefront. Connes et al. (1987) have proposed to recycle an old parabolic radio dish into a base to mount a large number of small telescopes linked by single-mode fibers to a beam combining station. It was shown (Connes and Reynaud 1988, Reynaud et al. 1992) that, despite the vibrations of the mount, the residual fluctuations of the optical path could be maintained at $\lambda / 20 \mathrm{RMS}$ through a servo control of the fiber length. The system involves both a thermal control of the waveguide and a fast servo with part of the fiber wrapped on a piezo cylinder. Pathlength equalization has also been investigated by Burnett et al (1991).

\section{Coupling starlight}

Starlight injection into a single-mode waveguide suffers from the fundamental incompatibility between a plane wave and a quasi-Gaussian guided beam.

According to the guided wave theory, if $\mathbf{E}_{\text {tel }}$ is the complex amplitude of the transverse component of the electric field at the telescope focus, and $\mathbf{E}_{\mathrm{fib}}$ the amplitude of the guided electric field, a good coupling occurs with a single-mode fiber when the overlap integral is maximized (Barell 1979, Neumann 1988):

$$
\eta=\left|\frac{n}{Z_{0}} \int \mathbf{E}_{\mathrm{tel}} \cdot \mathbf{E}_{\mathrm{fib}}^{*}\right|^{2}
$$

where $n$ is the waveguide refractive index, and $Z_{0}=377 \Omega$ the wave impedance of vacuum; integration is performed over the whole surface of the fiber front end. This means that the complex amplitude profile (and not the intensity) of the electric field at the focus of the telescope has to match well the complex amplitude mode profile.

In the ideal, diffraction limited case of an unobstructed pupil, the telescope produces an Airy pattern on the fiber head. The phase in the central disk is constant and, by a proper choice of the f-ratio, the size of the disk can be adjusted to the width of the guided beam. But the first ring, with a constant opposite phase, contributes negatively to the overlap integral and the net result is a maximum coupling efficiency of only $78 \%$. The presence of a central obstruction in the pupil is 


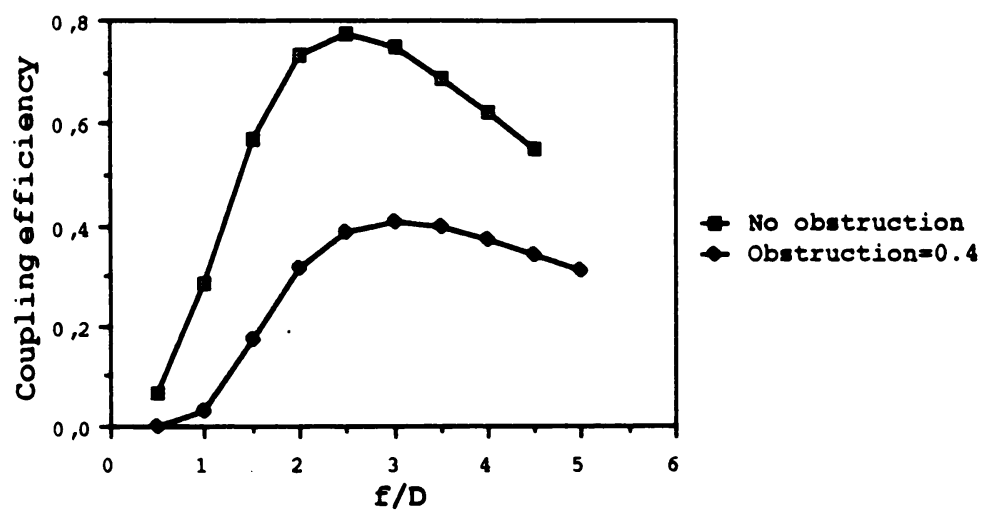

Fig. 2. Coupling efficiency vs. input beam f-ratio into a single-mode fiber $(N A=0.23$ and $V=2.1$ ) in a diffraction limited case. Top curve: no central obstruction; bottom curve: standard telescope with a central obstruction of $40 \%$ in diameter.

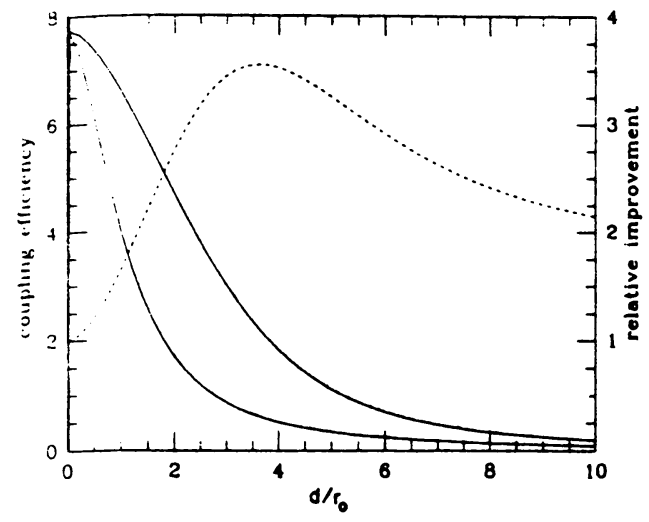

Fig. 3. Average coupling efficiency vs. seeing for a single-mode fiber at $V=2.2$. The upper curve is the image stabilization case; the lower curve is for no stabilization. The dashed curve goes with the scale on the right and is the ratio of the two solid curves (from Shaklan and Roddier (1988))

very important, because it reinforces the first ring of the Airy disk and significantly decreases the coupled power (Figure 2). In the case of a turbulent atmosphere, the single-mode fiber can extract only one coherence area from the corrugated wavefront, and the efficiency decreases with $D / r_{0}$ as the number of speckles increases (Shaklan and Roddier 1988). Image stabilization can improve the coupling by a factor of up to 3.5 ; when the image is stabilized, the total coupled power is maximum at $D / r_{0}=4$. Thus, $r_{0}$ being fixed by the atmospheric conditions, it can help when the seeing is bad to stop the telescope aperture so as to reach $D / r_{0}=4$. It is also found, as in the diffraction limited case, that the input f-ratio is not a critical parameter for optimum coupling. 

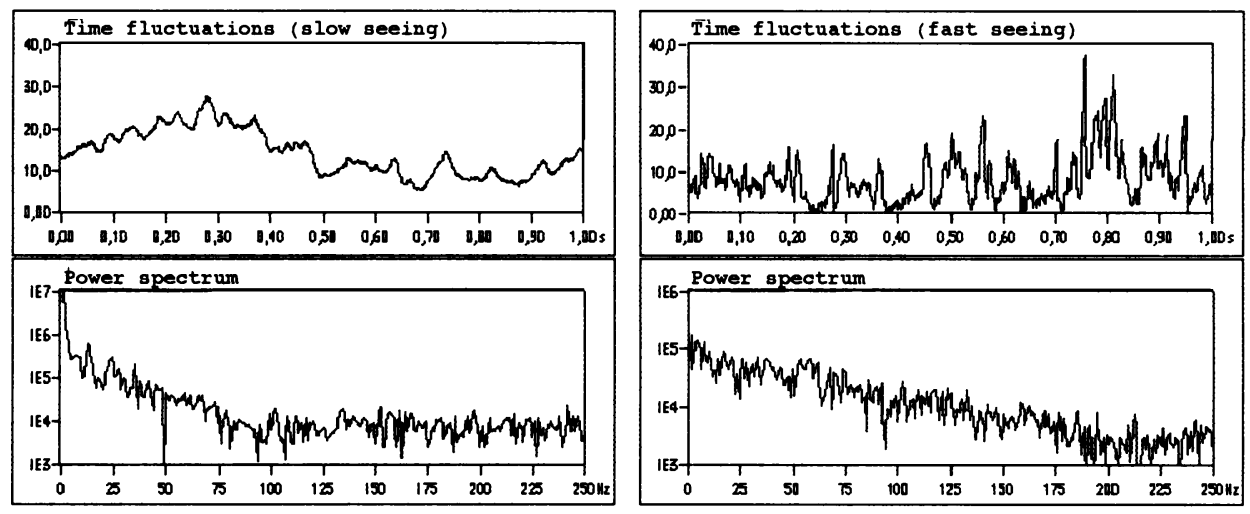

Fig. 4. Coupling fluctuations into a single-mode fiber in the infrared $\mathrm{K}$ band $(2 \mu \mathrm{m}<\lambda<$ $2.4 \mu \mathrm{m}$ ), with a $0.9 \mathrm{~m}$ pupil. Left: good (slow) seeing. Right: bad (fast) seeing.

Almost as important as the average coupling are its time fluctuations. Indeed, if one wants to take full advantage of the calibration capabilities offered by a singlemode waveguide that are described in Section 4, it is necessary at some point in the data reduction process to divide the interferogram by the coupling signals. A sudden drop in coupled power, even if it does not noticeably decrease the average coupling, locally destroys the signal to noise ratio and prevents the division. Coupling fluctuations depend not only on $r_{0}$ but also on seeing speed, as can be seen in Figure 4.

The most efficient way to increase coupling into a single-mode fiber is to improve the coherence on the pupil by using adaptive optics. The gain would be substantial even if the wavefront is only partially corrected. The intensity distribution in the focal plane of a telescope partially corrected by adaptive optics has been described as a "Bismarck profile", i.e. a coherent core surrounded by an incoherent halo. Not only the average coupling should be improved, but it is anticipated that the coherent core will provide a constant, non-zero DC component to the fluctuations and the local zeros will be avoided.

\section{Conclusion}

Integrated optics (fibers and couplers) offer a very attractive solution to most basic interferometric functions. The components are light, compact, rugged, and easy to assemble. There is nothing that guided optics can do that cannot be done with conventional optics, yet they can often perform it in a more elegant and efficient way. More specifically, the perfect spatial filtering provided by a single-mode waveguide and the possibility provided by couplers to easily extract calibration signals offer the possibility to improve the precision of fringe visibility measurement by an order of magnitude or more. 
The critical point is the injection of starlight. A single-mode waveguide can extract only one coherence area from a corrugated wavefront, and coupling efficiencies are best for long wavelengths and/or (at least partially) phased pupils. Fewer photons exit from a fiber interferometer: however, because of the spatial filtering, all of them can potentially contribute to the interferometric signal. The net result is that the total number of interferometric photons is roughly the same than in a traditional instrument (Shaklan et al. 1991).

Currently available integrated components are still far from perfect, but the margin for amelioration is large; in a sense, the situation is analog to what were integrated electronic components 40 years ago. Consequently, as their performances improve, it is expected that integrated optical devices will play an increasing role in stellar interferometry.

\section{References}

Barrell, K. F. and Pask, C: 1979, 'Optical fibre excitation by lenses', Optica Acta 26, 91-108

Burnett, J. G., Greenaway, A. H., Spowart, J., McBride, R. and Jones, J. D. C.: 1991, 'Balancing Optical Pathlengths in Fibre Interferometers', in High-resolution imaging by interferometry II, eds. Beckers, J. and Merkle, F. (ESO, Garching, Germany), 1287-1293

Burnett, J. G. and Jones, J. D. C.: 1992, 'Cutting optical fibers to equal lengths for optical broadband stellar interferometry', Appl. Opt. 31, 2977-2978

Burnett, J. G., Greenaway, A. H., Spowart, J., McBride, R. and Jones, J. D. C.: 1991, 'Balancing Optical Pathlengths in Fibre Interferometers', in High-resolution imaging by interferometry II, eds. Beckers, J. and Merkle, F. (ESO, Garching, Germany), 1287-1293

Connes, P., Froehly, C. and P. Facq: 1984, 'A Fiber-Linked Version of Project TRIO', in ESA Colloq. on Kilometric Optical Arrays in Space, eds. Longdon, N. and Melita, O. (Cargèse, Corsica), 49-61

Connes, P., Roddier, F., Shaklan, S. and Ribak, E.: 1987, 'Fiber-linked telescope arrays on the ground and in space' in ESA Workshop on Optical Interferometry in Space, ed. Volonté, S. (Granada, Spain), 73-83

Connes, P. and Reynaud, F.: 1988, 'Fiber tests on a radiotelescope', in High-resolution imaging by interferometry, ed. Merkle, F. (ESO, Garching, Germany), 1117-1129

Coudé du Foresto, V. and Mazé, G.: 1990, 'Using single-mode infrared fibers for interferometry: progress report', in SPIE Proc. 1237, 538-547

Coudé du Foresto, V. and Ridgway, S.: 1991, 'FLUOR: a stellar interferometer using single-mode infrared fibers', in High-resolution imaging by interferometry II, eds. Beckers, J. and Merkle, F. (ESO, Garching, Germany), 731-740

Coudé du Foresto, V., Ridgway, S. and Mariotti, J.-M.: 1992, in 'Integrated optics for an interferometric link', ESA Colloquium on Targets for Space-Based Interferometry, ed. Volonté, S. (Beaulieu, France), 219-224

Forman, P. R. and Jahoda, F. C.: 1988, 'Linear birefringence effects on fiber-optic current sensors', Appl. Opt. 27, 3088-3096

Froehly, C.: 1981, 'Coherence and interferometry through optical fibers', in Scientific Importance of High Angular Resolution at Infrared and Optical Wavelengths, eds. Ulrich, M. H. and Kjr, K. (ESO, Garching, Germany), 285-293

Gloge, D.: 1971, 'Weakly Guiding Fibers', Appl. Opt. 10, 2252-2258

Harmon, R. A.: 1982, 'Polarisation stability in long lengths of monomode fibre', Electron. Lett. 18, 1058-1060

Jinguji, K., Horiguchi, M., Shibata, S., Kanamori, T., Mitachi, S. and Manabe, T. : 1982, 'Material dispersion in fluoride glasses', Electron. Lett. 18, 164-165

Léna, P., Ridgway, S. T. and Mariotti, J. M.:1988, 'Interferometric beam recombination at infrared wavelengths', in High-resolution imaging by interferometry, ed. Merkle, F. (ESO, Garching, Germany), 1039-1055 
Mariotti, J.-M. and Ridgway, S. T.: 1988, 'Double Fourier spatio-spectral interferometry: combining high spectral and high spatial resolution in the near infrared', Astron. Astrophys. 195, 350-363

Monerie, M., Alard, F. and Mazé, G.: 1985, 'Fabrication and Characterisation of Fluoride-glass single-mode fibers', Electron. Lett. 21, 1179-1181

Neumann, E.-G.: 1988, Single-Mode Fibers, Springer-Verlag, Berlin

Noda, J., Okamoto, K. and Sasaki, Y.: 1986, 'Polarization-Maintaining Fibers and Their Applications', Journal of Lightwave Technology LT-4, 1071-1089

Payne, D., Barlow, A. and Hansen, J.: 1982, 'Developement of Low- and High-Birefringence Optical Fibers', IEEE J. Quant. Elec. QE-18, 477-487

Ramaswamy, V., Standley, R. D., Sze, D. and French, W. G.: 1978, 'Polarization Effects in Short Length, Single Mode Fibers', The Bell System Technical Journal 57, 635-651

Reynaud, F., Alleman, J. J. and Connes, P.: 1992, 'Interferometric control of fiber lengths for a coherent telescope array', App. Opt. 31, 3736-3743

Rohloff, R.-R. and Leinert, C.:1991, 'A fiber optics x-coupler as phase tracker in an astronomical interferometer - concept and first laboratory measurements', in High-resolution imaging by interferometry II, eds. Beckers, J. and Merkle, F. (ESO, Garching, Germany), 1303-1312

Ross, J. N.: 1984, 'The rotation of the polarization in low birefringence monomode optical fibres due to geometric effects', Optical and Quantum Electronics 16, 455-461

Shaklan, S. B. and Roddier, F.: 1987, 'Single-mode fiber optics in a long-baseline interferometer', Appl. Opt. 26, 2159-2163

Shaklan, S. and Roddier, F.: 1988, 'Coupling starlight into single-mode fiber optics', Appl. Opt. 27, 2334-2338

Shaklan, S. B.: 1989, Multiple Beam Correlation Using Single-Mode Fiber Optics with Application to Interferometric Imaging, PhD thesis, The University of Arizona, Tucson

Shaklan, S., Reynaud, F. and Froehly, C.: 1990, 'Multimode Fiber Optics Broad Spectral Band Interferometer', in SPIE Proc. 1319, 448-449

Shaklan, S., Colavita, M. and Shao, M.: 1991, 'Visibility Calibration Using Single-Mode Fibers in a Long-Baseline interferometer', in High-resolution imaging by interferometry II, eds. Beckers, J. and Merkle, F. (ESO, Garching, Germany)

Sheen, S. K. and Giallorenzi, T. G.: 1979, 'Single-mode fiber multiterminal star directional coupler', Appl. Phys. Lett. 35, 131-133

Sheen, S. K.: 1980, 'Fiber-optic gyroscope with $3 \times 3$ directional coupler', Appl. Phys. Lett. 37, $869-871$

Tekippe, V. J. and Willson, W. R.: 1985, 'Single-mode directionnal couplers', Laser Focus 21, 132-144

Ulrich, R., Rashleigh, S. C. and Eickhoff, W.: 1980, 'Bending induced birefringence in single-mode fibers', Opt. Lett. 5, 273-275

Wang, C. C., Burns, W. K. and Villaruel, C. A.: 1985, ' $9 \times 9$ single-mode fiber-optic star couplers', Opt. Lett. 10, 49-51

Wysocki, J. A.:1987, 'Mechanical properties of IR glasses and fibers', in SPIE Proc. 843, 21-33

Zhao, P., Mariotti, J.-M., Léna, P. and Coudé du Foresto, V.: 1992, 'IR single-mode fiber optics for double Fourier interferometry', in ESA Colloquium on Targets for Space-Based Interferometry, ed. Volonté, S. (Beaulieu, France), 225-230 